\title{
A genetic handicap approach: how to estimate the genome-wide burden of slightly-deleterious variants in a model population
}

\author{
Victor Shamanskiy \\ Center for Mitochondrial Functional \\ Genomics \\ Immanuel Kant Baltic Federal \\ University \\ Kaliningrad, Russia \\ v.a.shamanskiy@gmail.com
}

\author{
Konstantin Gunbin \\ Institute of Cytology and Genetics SB \\ RAS, Novosibirsk, Russia \\ Immanuel Kant Baltic Federal \\ University \\ Kaliningrad, Russia \\ genkvg@gmail.com
}

\author{
Konstantin Popadin \\ School of Life Sciences \\ Ecole Polytechnique Federale de \\ Lausanne, Lausanne, Switzerland \\ Center for Mitochondrial Functional \\ Genomics, Immanuel Kant Baltic \\ Federal University \\ Kaliningrad, Russia \\ konstantinpopadin@gmail.com
}

\begin{abstract}
We hypothesized that strongly-deleterious mutation (hereafter 'genetic handicap'), epistatically interacting with the burden of slightly-deleterious variants (SDV), segregating in the genome might be of great importance as a marker of the burden. We expect that handicap-carriers (individuals surviving with the genetic handicap) have decreased burden of SDV as compared to controls. Here to test this hypothesis under different parameter space (mutation rate, severity of SDV and severity of the genetic handicap, effective population size) we use SLim simulation environment. We conclude that the genetic handicap hypothesis indeed works within a broad spectrum of biologically meaningful parameters.
\end{abstract} SLiM

Keywords - SDVs, epistatic interactions, genetic handicap,

Introduction

All organisms harbor numerous slightly-deleterious variants (SDVs) in their genomes. However, the composition of SDVs, as well as its total effect on fitness, are poorly known due to extreme complexity of the system: thousands of SDVs with very small individual phenotypic effects can interact with each other in various ways and with environment in non-additive manner, making reconstruction of fitness space from genome data almost impossible.

Taking into account widespread negative epistatic interactions between SDVs [1] we introduced a genetic handicap hypothesis: the healthy carrier of a severelydeleterious variant (hereafter "genetic handicap") is expected to have a decreased number of SDVs in genome compared with the organisms without genetic handicap. If so, the genetic handicap can be used in evolutionary and population studies as a signature of genome clearance.

Recently, considering trisomy 21 as a genetic handicap in humans [2] we got the first empirical evidence, supporting our hypothesis: live-born Down Syndrome individuals have decreased the number of SDVs compared to control individuals. Here we computationally investigate the genetic handicap hypothesis in more detail.

\section{Genetic handicap hypothesis}

If the biological fitness of an organism is determined as a function of its mutational load, and this mutational load consists mainly of unconditionally deleterious mutations, then we expect to observe a trade-off between the presence of a severely deleterious mutation and the total number of SDVs. Using the term handicap, introduced by Amotz Zahavi as a marker of high genome quality of a carrier [3], and defined in Oxford dictionary as "a circumstance that makes progress or success difficult", we introduce a genetic handicap principle, in which an organism bearing a severely-deleterious mutation (hereafter a 'genetic handicap' in Zahavi's sense) is only fit (viable at birth) if its genome wide load of SDVs is sufficiently low. The rationale for this hypothesis is that only highly fit organisms, with a sufficiently low SDV load, are able to tolerate the effects of severely-deleterious mutations and survive. It is interesting to note, that our approach resembles the 'liability' introduced by Falconer as a single continuous normally distributed factor representing a mixture of environmental and genetic traits and determining a probability to get a complex disease [4].

Here, we define a genetic handicap as a severelydeleterious mutation with very broad effect on cellular metabolism. The broad effect assumes that the genetic handicap is unlikely to be compensated / modified by a few conditional variants and therefore, might only be compensated by a genome-wide decreased load of unconditionally deleterious variants. In this case, we can approximate the total mutational load using the load of unconditionally deleterious mutations.

We assume here that embryonic viability is an important component of fitness, so that the genetic handicap splits the affected population into two groups: survivors at birth and non-survivors, thus providing a simple grouping of affected organisms based on their SDV loads. The main prediction of this hypothesis is that handicap-carriers have a lower number of SDVs as compared to controls (live-born organisms without handicap). Moreover, the stronger the genetic handicap severity, the higher the difference in loads of SDVs between handicap-carriers and controls is expected.

\section{The genetic handicap model}

The genetic handicap model is shown on the Fig 1. (A) The distributions of the number of SDVs in control (gray) and affected (red) populations are demonstrated. The genetic handicap mutation (black arrow) is a numerical equivalent of many SDVs. (B) Truncation selection eliminates all organisms with the number of SDVs higher than the given threshold (vertical black line) from both control and affected populations. (C) Handicap carriers have 
a decreased number of SDVs (SDVs do not include the genetic handicap per se) compared to controls; this difference represents the handicap effect.

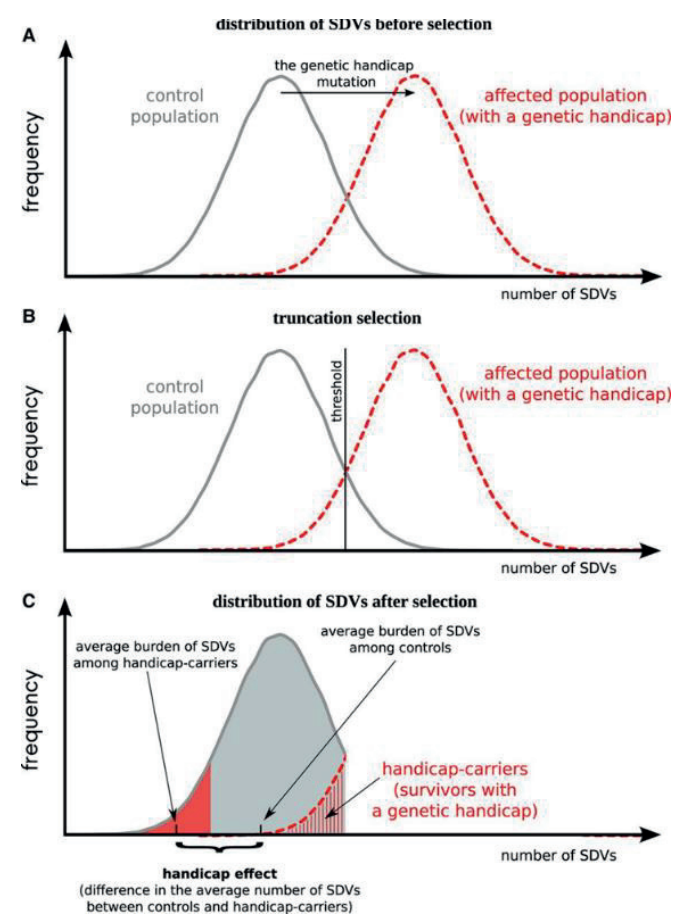

Fig. 1. The genetic handicap model

Forward based simulation using SLIM

SLiM (https://messerlab.org/slim/ ) is an evolutionary simulation framework that combines a powerful engine for population genetic simulations with the capability of modeling arbitrarily complex evolutionary scenarios. Simulations are configured via the integrated Eidos scripting language that allows interactive control over practically every aspect of the simulated evolutionary scenarios. The underlying individual-based simulation engine is highly optimized to enable modeling of entire chromosomes in large populations.

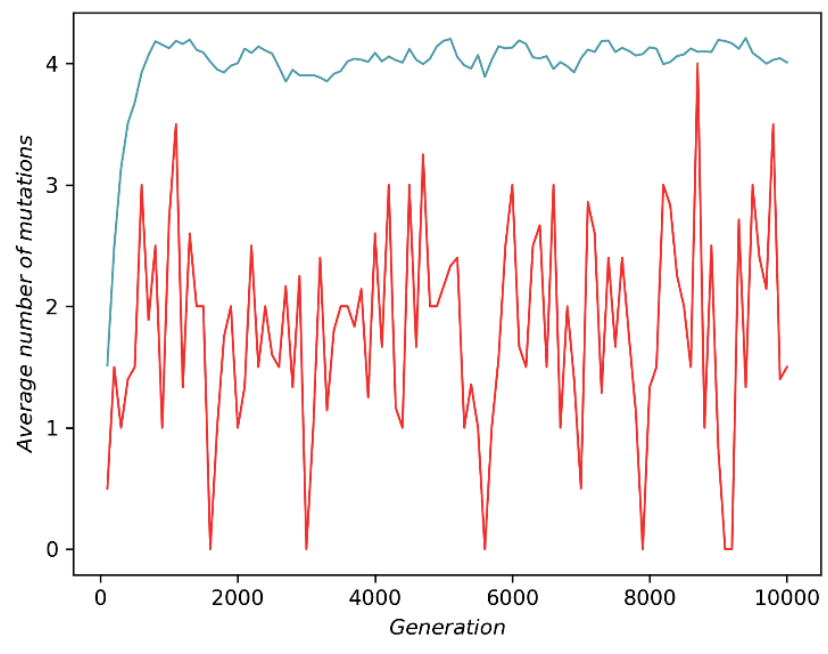

Fig. 2. The Mutation-Selective Balance with handicap-carriers

To test the genetic handicap hypothesis in the SLiM environment we followed two main steps. First of all we established numerous populations under mutation-selection equilibrium (MSE). One example of such a population (effective population size $=100000$, genome length $=$ 10000 , selection coefficient of each SDV is 0.01 , mutation rate of SDV per genome per generation is 0.000001) is provided in Fig 2, where the blue line reflects - how the average number of SDV per genome of average organism (axis $\mathrm{Y}$ ) is changing with generations (axis X). We can see that the population reaches the MSE during the first 1000 generations. Second, we introduced an additional category of a mutation - the genetic handicap (coefficient of selection $=0.5$, mutation rate $=0.000000001)$ and plotted the burden of SDV among handicap carriers (organisms surviving with the genetic handicap mutation) as red line (Fig 2 ) We can see that despite strong fluctuations, red line is located effectively below the blue line, confirming that handicap carriers indeed have decreased burden of SDV. (Fig. 2).

\section{Acknowledgment}

This work is supported by the 5 Top 100 Russian Academic Excellence Project at the Immanuel Kant Baltic Federal University, and by the Russian Foundation for Basic Research grant 18-29-13055.

\section{References}

[1] M. Sohail, "Negative selection in humans and fruit flies involves synergistic epistasis." Science. (2017): 539-542;

[2] K. Popadin et al, "Slightly deleterious genomic variants and transcriptome perturbations in Down syndrome embryonic selection", Genome Res. 28 (2018): 1-10;

[3] A. Zahavi, "Mate selection-A selection for a handicap", Journal of Theoretical Biology 53 (1975): 205-214;

[4] D. S. Falconer, "The inheritance of liability to certain diseases, estimated from the incidence among relatives", Annals of human genetics 29 (1965): 51-76. 